\title{
STUDY OF INTERFERENCE EFFECTS ON THE $\Lambda$ (1405) LINE SHAPE MEASURED BY HADES IN p+p COLLISIONS*
}

\author{
L. FABBIETTI \\ Excellence Cluster "Universe”, Technische Universität München, \\ Boltzmannstr. 285478 Garching, München, Germany \\ laura.fabbietti@ph.tum.de
}

Published 14 January 2014

\begin{abstract}
In this work we investigate the possible interference effects between the $\Lambda(1405)$ and the non-resonant final state in $\mathrm{p}+\mathrm{p}$ reactions at $3.5 \mathrm{GeV}$ beam kinetic energy measured by the HADES collaboration. The two poles nature of the $\Lambda(1405)$, which is supported by most of the theoretical models, are parametrized within a simplified phenomenological model with Breit-Wigner functions to evaluate the interference effects. The model is adjusted such as to be in accordance with the theoretical prediction for the $\Lambda(1405)$ in $\mathrm{p}+\mathrm{p}$ collisions.
\end{abstract}

\section{Introduction}

Recently, the HADES collaboration has measured $\Lambda(1405)$ production in protonproton reactions at a beam kinetic energy of $3.5 \mathrm{GeV},{ }^{1}$ where the $\Lambda(1405)$ hyperon has been reconstructed in the charged $\Sigma^{ \pm} \pi^{\mp}$ decay channels. By investigating the $\Sigma \pi$ invariant mass distributions, clear peak structures below $1400 \mathrm{MeV} / \mathrm{c}^{2}$ were observed. These structures were interpreted by a small contribution of $\Sigma(1385)^{0}$ and a large contribution of a low mass $\Lambda(1405)$ signal (see also ${ }^{2}$ ). The experimental data were finally described by an incoherent sum of Monte Carlo simulations, where the $\Lambda(1405)$ was simulated to follow a Breit-Wigner type distribution with a BreitWigner mass of $1385 \mathrm{MeV} / \mathrm{c}^{2}$ and a width of $50 \mathrm{MeV} / \mathrm{c}^{2}$.

The experimental data, where the maximum of the $\Sigma \pi$ missing mass lies below the nominal value of $1405 \mathrm{MeV} / \mathrm{c}^{2}$ associated to the $\Lambda(1405)$, suggest a shift of this resonance towards lower masses. In this paper we want to address different possible explanations for the observed mass shift of the $\Lambda(1405)$ in the new HADES data and aim to stimulate theorists to further investigate the production of this resonance in $\mathrm{p}+\mathrm{p}$ reactions. From the theory side, the authors of ${ }^{3}$ followed a unitarized

* This is an Open Access article published by World Scientific Publishing Company. It is distributed under the terms of the Creative Commons Attribution 3.0 (CC-BY) License. Further distribution of this work is permitted, provided the original work is properly cited. 
coupled channel approach based on the chiral Lagrangian in order to predict the $\Lambda(1405)$ line shape in $\mathrm{p}+\mathrm{p}$ reactions for the $\Sigma^{0} \pi^{0}$ decay channel. In their Ansatz the $\Lambda(1405)$ was generated from pion, kaon and rho meson exchange mechanisms, all of them leading to a different coupling to the two $\Lambda(1405)$ poles. The coherent sum of all contributions results in a $\Lambda(1405)$ line shape with a maximum in the $\Sigma \pi$ mass distribution at around $1410 \mathrm{MeV} / \mathrm{c}^{2}$, result which is compatible with the $\Lambda$ (1405) signal measured by the ANKE collaboration in the $\Sigma^{0} \pi^{0}$ decay channel. ${ }^{4}$ This calculation is the only one available for $\mathrm{p}+\mathrm{p}$ reactions but since the HADES data refer to the charged decay channels a quantitative comparison results difficult. Nevertheless, the results by ${ }^{3}$ have been used in this work as a starting point to model the $\Lambda(1405)$ as the combination of two Breit-Wigner functions.

In general, the HADES data show a larger contribution by the non-resonant $\Sigma \pi$ production in comparison with ANKE. In particular, it has been shown in ${ }^{5}$ that the $\Delta^{++}$couples strongly to the $\Sigma^{-} \pi^{+} p K^{+}$final state via the reaction $p+p \rightarrow$ $\Sigma^{-}+\Delta^{++}+K^{+}$and it cannot be excluded that $N^{*} / \Delta^{0}$ resonances contribute to the $\Sigma^{+} \pi^{-} p K^{+}$channel as well. These contributions might appear in the $\mathrm{I}=0$ channels and hence interfere with the resonant amplitude.

\section{Influence of Interference Effects}

Interference between the resonant and the non-resonant amplitudes can affect the observed mass distribution considerably. In order to evaluate this scenario, we take the result of the chiral Ansatz ${ }^{3}$ as a starting point to develop a simple model, which we use to parametrize the $\Lambda(1405)$ amplitude and to interpret the HADES data. The predicted $\Sigma \pi$ spectrum of ${ }^{3}$ is shown in the gray band of Fig. 1, with a peak at $1410 \mathrm{MeV}$ and with the typical $\Lambda(1405)$ shape, having a sharp drop at the $\bar{K} N$

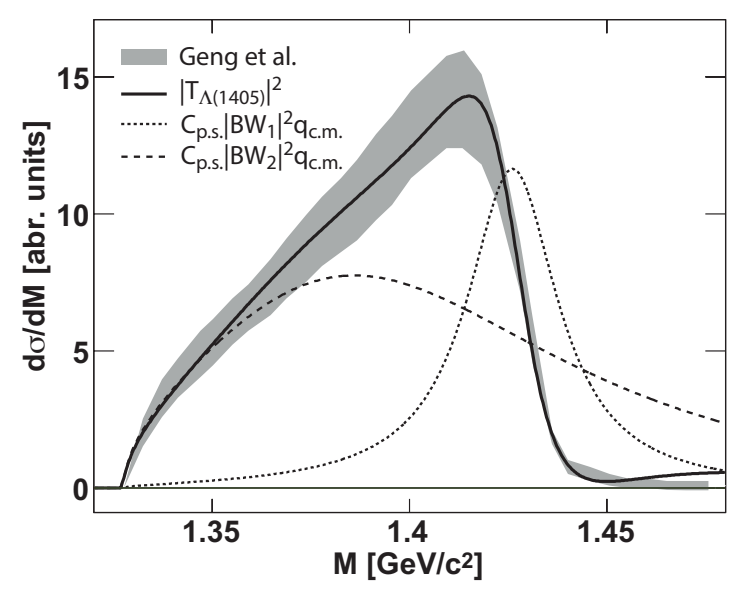

Fig. 1. The $\Lambda(1405)$ spectral shape calculated within effective field theory together with the result from the Breit-Wigner parametrisation. See text for details. 
threshold. The idea is to reconstruct this spectrum by a coherent sum of two BreitWigner functions (BW), where each of these BW amplitudes represents one of the two $\Lambda(1405)$ poles. Within this approach the total $\Lambda(1405)$ amplitude reads then as follows:

$$
\begin{aligned}
\frac{d \sigma}{d m}= & \left|T_{\Lambda(1405)}\right|^{2}= \\
= & C_{p . s .}(m)\left|B W_{1}(m) e^{i \varphi_{1}}+B W_{2}(m)\right|^{2} q_{c . m .} \\
& \quad \text { with } B W_{i}=A_{i} \frac{1}{\left(m-m_{0, i}\right)^{2}+i m_{0, i} \Gamma_{0, i}}
\end{aligned}
$$

$C_{p . s .}(m)$ is a dimensionless weight function, normalized to unity in the mass range of $1280-1730 \mathrm{MeV} / \mathrm{c}^{2}$. This function considers the limited production phase space of the $\Lambda(1405)$ in $p+p$ reactions. $q_{c . m}$. is the decay momentum of $\Sigma$ and $\pi$ in the $\Lambda(1405)$ rest frame (in units of $[\mathrm{MeV} / \mathrm{c}]$ ). The Breit-Wigner function is a simple relativistic parameterization with amplitude $A_{i}$ in units of $\left[\sqrt{\mu b / \mathrm{c}} \cdot \mathrm{MeV} / \mathrm{c}^{2}\right]$, mass $m_{0, i}$ and width $\Gamma_{0, i}$ in units of $\left[\mathrm{MeV} / \mathrm{c}^{2}\right]$. Thus, the whole expression has dimensions of $\left[\frac{\mu b}{\mathrm{MeV} / \mathrm{c}^{2}}\right]$. We also introduce a free phase $e^{i \varphi_{1}}$, which determines the interference between the two Breit-Wigner functions. Furthermore, we make use of the recent coupled channel calculations by Ikeda et al. ${ }^{6}$ which are constrained by the new SIDDARTHA data on kaonic hydrogen. ${ }^{7}$ In this work the $\bar{K} N$ pole was found at $z_{1}=1424_{-23}^{+7}+i 26_{-14}^{+3} \mathrm{MeV}$, while the $\Sigma \pi$ pole was found at $z_{2}=1381_{-6}^{+18}+$ $i 81_{-8}^{+19} \mathrm{MeV}$. With these values we constrain the Breit-Wigner mass $m_{i} c^{2}=\operatorname{Re}\left(z_{i}\right)$ and the Breit-Wigner width $\Gamma_{0, i} c^{2}=2 \operatorname{Im}\left(z_{i}\right)$ of our model to vary only within the given ranges.

Although the parameterization of equation (1) is simplified compared to the advanced calculations in, ${ }^{3}$ it still allows us to reconstruct the spectral shape in Fig. 1 (gray band). By fitting the equation (1) to the theoretical prediction, we obtain the black distribution with the fit parameters listed in Table 1. This distribution is consistent with the gray band in Fig. 1. Especially the peak structure around $1410 \mathrm{MeV} / \mathrm{c}^{2}$ and the drop at the $\bar{K} N$ threshold are reproduced correctly. Additionally included in the figure are the absolute contributions of the two BreitWigner functions $C_{p . s .}(m)\left|B W_{1}(m)\right|^{2} q_{c . m \text {. }}$ and $C_{p . s .}(m)\left|B W_{2}(m)\right|^{2} q_{c . m \text {. }}$ (dotted and dashed lines).

In this way we have fixed the parameterization of the $\Lambda(1405)$ and can now study the maximal interference effects with the non-resonant background. For this

Table 1. Table with fit parameters obtained by fitting equation (1) to the theoretical prediction shown in Fig. 1.

\begin{tabular}{cccccc}
$m_{1}$ & $\Gamma_{1}$ & $m_{2}$ & $\Gamma_{2}$ & $A_{1} / A_{2}$ & $\varphi_{1}$ \\
\hline 1426 & 28 & 1375 & 147 & 0.23 & $205^{\circ}$
\end{tabular}


purpose, we fit the HADES results for the $\Sigma^{+} \pi^{-}$and $\Sigma^{-} \pi^{+}$invariant mass distributions simultaneously with the following two functions:

$$
\begin{aligned}
& \left(\frac{d \sigma}{d m}\right)_{\Sigma^{+} \pi^{-}}=\left|A_{\Lambda(1405)} T_{\Lambda(1405)}+e^{i \alpha} A_{\Sigma^{+} \pi^{-}} T_{\Sigma^{+} \pi^{-}}\right|^{2}+\left|B W_{\Sigma(1385)^{0}}\right|^{2}+\left|B W_{\Lambda(1520)}\right|^{2} \\
& \left(\frac{d \sigma}{d m}\right)_{\Sigma^{-} \pi^{+}}=\left|A_{\Lambda(1405)} T_{\Lambda(1405)}+e^{i \beta} A_{\Sigma^{-} \pi^{+}} T_{\Sigma^{-} \pi^{+}}\right|^{2}+\left|B W_{\Sigma_{(1385)^{0}}}\right|^{2}+\left|B W_{\Lambda(1520)}\right|^{2}
\end{aligned}
$$

The contributions from $\Sigma(1385)^{0}$ and $\Lambda(1520)$ are parameterized as Breit-Wigner functions so that they match the extracted shapes and yields reported in. ${ }^{1}$ We assume here that they do not interfere with the other contributions to the $\Sigma \pi$ invariant mass spectra and thus add them incoherently in the equations (2) and (3).

The $\Lambda(1405)$ is parameterized as described above. $A_{\Lambda(1405)}$ is a free fit parameter which determines the absolute yield of $\Lambda(1405)$.

The non-resonant background shapes for the $\Sigma^{+} \pi^{-}$and $\Sigma^{-} \pi^{+}$channels $\left(T_{\Sigma^{+} \pi^{-}}\right.$ and $T_{\Sigma^{-} \pi^{+}}$) are described by modified polynomials of 4 th order, which read as follows:

$$
T_{\Sigma \pi}(m)=\left[C_{p . s .}(m) q_{c . m .} \sum_{n=0}^{4} a_{n} m^{n}\right]^{\frac{1}{2}}
$$

This parameterization has no physical meaning but it was chosen such as to describe the simulated $\Sigma \pi$ invariant mass distributions in. ${ }^{1}$ The parameters $a_{n}$ are given in units of $\left[\left(\mathrm{MeV} / \mathrm{c}^{2}\right)^{-n}\right]$ and their values are listed in Table 2 . The modified polynomials are multiplied by constant factors $A_{\Sigma^{+} \pi^{-}}$and $A_{\Sigma^{-} \pi^{+}}$(see equation (2) and (3)), which have dimensions of $\left[\frac{\sqrt{\mu b / \mathrm{c}}}{\mathrm{MeV} / \mathrm{c}^{2}}\right]$. These are again free fit parameters, determining the absolute yields of the non-resonant channels, where a value of $A_{\Sigma \pi}=1$ corresponds to the yield extracted in. ${ }^{1}$ Furthermore, complex phases $e^{i \alpha}$ and $e^{i \beta}$ have been included so that the modeled background can interfere with the $\Lambda(1405)$ amplitude. The values of these phases are determined by the fitting procedure as

Table 2. Table with coefficients for the description of the non-resonant background according to equation (4).

\begin{tabular}{cccc}
$T_{\Sigma \pi}$ & $a_{0}$ & $a_{1}$ & $a_{2}$ \\
\hline$T_{\Sigma^{+} \pi^{-}}$ & $7.949 \cdot 10^{-3}$ & $-4.412 \cdot 10^{-7}$ & $-1.558 \cdot 10^{-9}$ \\
$T_{\Sigma^{-} \pi^{+}}$ & $-2.387 \cdot 10^{0}$ & $2.512 \cdot 10^{-3}$ & $1.315 \cdot 10^{-6}$ \\
\hline
\end{tabular}

\begin{tabular}{ccc}
$T_{\Sigma \pi}$ & $a_{3}$ & $a_{4}$ \\
\hline$T_{\Sigma^{+} \pi^{-}}$ & $2.942 \cdot 10^{-12}$ & $-1.121 \cdot 10^{-15}$ \\
$T_{\Sigma^{-} \pi^{+}}$ & $-2.254 \cdot 10^{-9}$ & $6.481 \cdot 10^{-13}$
\end{tabular}


Table 3. Obtained fit parameters after fitting equations (2) and (3) to the experimental data points in Fig. 2.

\begin{tabular}{ccccc}
$A_{\Lambda(1405)}$ & $A_{\Sigma^{+} \pi^{-}}$ & $A_{\Sigma^{-} \pi^{+}}$ & $\alpha$ & $\beta$ \\
\hline 1.06 & 0.93 & 1.04 & $67^{\circ}$ & $109^{\circ}$
\end{tabular}

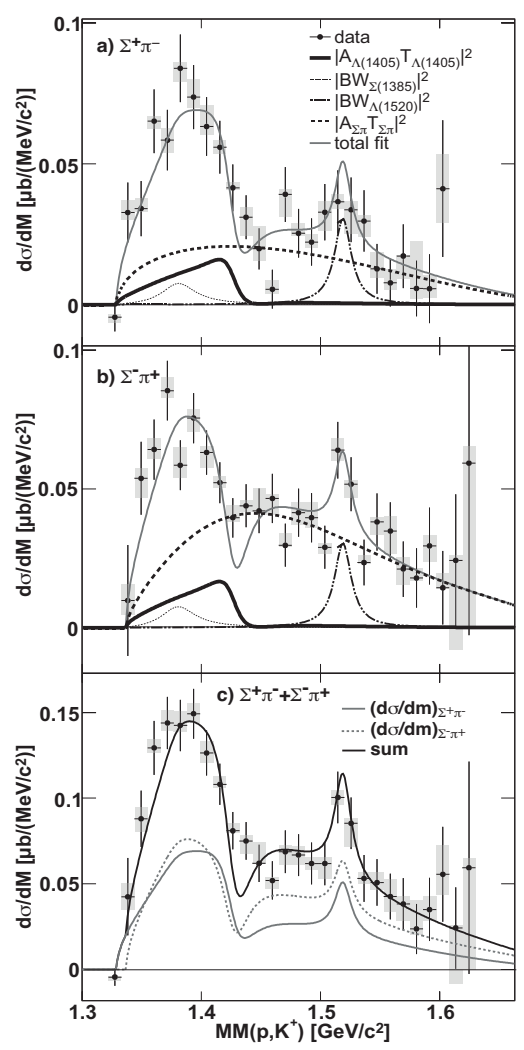

Fig. 2. Missing mass spectrum to proton and $K^{+}$. The black data points are the HADES measurements, for the $\Lambda(1405)$ in the $\Sigma^{+} \pi^{-}$(a) and $\Sigma^{-} \pi^{+}$(b) decay channel. Panel c) shows the summed spectrum of a) and b). The black lines in a) and b) are the results from the simultaneous fit with equation (2) and (3), the gray line represents the sum of all fitted functions. In c) the fit functions corresponding to the $\Sigma^{+} \pi^{-}$and $\Sigma^{-} \pi^{+}$channels respectively are shown in gray and the sum of both functions is shown in the black line.

well. Hence, the simultaneous fit of the two functions (2) and (3) to the experimentally determined $\Sigma^{+} \pi^{-}$and $\Sigma^{-} \pi^{+}$invariant mass distributions is characterized by five free parameters.

We consider here a scenario of maximal interference, which means that the whole non-resonant background interferes with the $\Lambda(1405)$ amplitude. The best results of the fitting procedure (gray lines) are illustrated together with the experimental data in Fig. 2 panel a) and b). The black lines show the amplitude of the $\Lambda(1405)$, 
the red lines the contribution by the non-resonant $\Sigma \pi$ channels and the gray lines correspond to the coherent sum of all contributions. The fit parameters are listed in Table 3. A reasonable description of the data is achieved, expressed in a normalized $\chi^{2}$ value of 1.23 . In panel c) of Fig. 2 we show the sum of the two invariant mass distributions and compare it to the sum of our model (black line).

The main message of the three pictures is that, assuming a maximal interference between the $\Lambda(1405)$ and the non-resonant contributions and the large phases given in Table 3, one obtains a shift of the maximum to lower masses. This is the case although the mass maximum of the initial $\Lambda(1405)$ amplitude is located around $1400 \mathrm{MeV} / \mathrm{c}^{2}$. By integrating $\left|A_{\Lambda(1405)} T_{\Lambda(1405)}\right|^{2}$ (black lines in panel a) and b)), the total cross section of the reaction $p+p \rightarrow \Lambda(1405)+p+K^{+}$is determined to be $3.3 \mu b$. This is considerably smaller than the value extracted in, ${ }^{1}$ where an incoherent approach was used with a low mass $\Lambda(1405)$ signal.

\section{Summary}

One possible explanation for the observed mass shift is based on interference effects. For that purpose we have developed a simple model, where we assumed the $\Lambda(1405)$ to consist of two poles, parameterized as Breit-Wigner amplitudes. The contribution and position of these poles were first chosen such as to match the $\Lambda(1405)$ line shape of $^{3}$ with the mass peak appearing at $\approx 1410 \mathrm{MeV} / \mathrm{c}^{2}$. With this model we could show that maximum interference between the $\Lambda(1405)$ amplitude and the non-resonant $\Sigma \pi$ background can indeed create a low mass signal and thus describe the HADES data. However, it was argued that this explanation is probably very unrealistic, since no comparable mass shifts have been observed in the spectral shape of the $\Sigma(1385)^{+}$in the $\Lambda-$ p final state $^{8}$ or for the $\Lambda(1520)$ in the $\Sigma \pi$ decay channel. Moreover, it seems to be rather peculiar that interferences between the $\Lambda(1405)$ and the non-resonant background should result in the same mass shift for both, the $\Sigma^{+} \pi^{-}$and the $\Sigma^{-} \pi^{+}$invariant mass distributions, where in both cases the physical origin of the non-resonant background is quite different. This suggests that more precise calculations, including all contributing channels, are probably needed to describe the $\Lambda(1405)$ spectral function extracted from the charged decays in $\mathrm{p}+\mathrm{p}$ collisions.

\section{References}

1. HADES Collab. ( G. Agakishiev et al.), Phys. Rev. C87, 2025201 (2013).

2. HADES Collab. ( G. Agakishiev et al.), Hyperfine Interact.210,(2012), p 45-51.

3. L.S. Geng and E. Oset, Eur.Phys.J. A34, (2007), p 405-412.

4. ANKE Collab. (I. Zychor et al.), Phys. Lett B660, (2008), p 167-171.

5. HADES Collab. ( G. Agakishiev et al.), Nucl. Phys. A881, (2012), p 178-186.

6. Y. Ikeda, T. Hyodo and W. Weise, Nucl. Phys. A881,(2012),p 98-114.

7. SIDDHARTA Collab. (M. Bazzi et al.), Phys. Lett B704,(2011), p. 113-117.

8. HADES Collab. ( G. Agakishiev et al.), Phys. Rev. C85, 035203 (2012). 